\title{
Improved Weiner Filter Used in Speech Enhancement Jian Peng ${ }^{1, a}$, Fei $\mathbf{Q}^{2, b}$ \\ ${ }^{1}$ WuHan City, Hubei Province, China \\ ${ }^{2}$ WuHan City, Hubei Province, China \\ aqixinhai@163.com, b809847300@qq.com
}

Keywords: Speech enhancement, Weiner filter, Iterated weiner filter

\begin{abstract}
The aim of speech enhancement is to extract pure primary speech from the signals with noises as much as possible, to eliminate the background noises, to improve the sound quality and to improve the articulation, intelligibility and comfort degree of the speech. In order to minimize the residual noise and background noise as much as possible, realized that the improved Weiner filter is applied in speech enhancement based on the basic Weiner filter, which effectively suppresses the "musical noise" and improves the quality of hearing speech, making the speech enhancement effect more satisfactory.
\end{abstract}

\section{Introduction}

Speech signal processing ${ }^{[1]}$ has been a hot research spot for local and foreign rea researchers in the information era, which involves Acoustics, signal processing, bionics. But there is always noise from the environment when passing the information, which makes the gets not the originals. So we must add speech enhancement system ${ }^{[2]}$, or suppressing background noise to improve the quality of voice communications. We designed an improved Weiner filter enhancement method on the based of original Weiner filter enhancement method, whose goal is improving the comfort of speech but not improving the signal to noise ratio. At last, we conduct a simulation experiment by Matlab.

\section{Weiner filter speech enhancement system}

\subsection{Weiner filter and its basic ideas}

What the Weiner filtering method ${ }^{[3]}$ do is collecting noise and noisy speech signal subtracting from the noisy speech amplitude spectrum after the Wiener filter through the noise component amplitude spectrum, then adding the phase noise of the speech spectrum band, inversing Fourier transform to get enhanced voice signal.

\subsection{Weiner filter speech enhancement algorithm}

For noisy speech signal $y(t)=s(t)+d(t), s(t)$ is Clean speech signal, $d(t)$ is noise signal.We should determine the impulse response $h(t)$ to make sure that the $E\left[\left|s^{\prime}(t)-s(t)\right|^{2}\right]$ is the smallest.

Assumpting $s(t)$ and $d(t)$ are short-stationary random process, by Weiner-Hopf integral equation ${ }^{[4]}$ : we can get

$$
R_{s y}(\tau)=\int_{-\infty}^{+\infty} h(\alpha) R_{y y}(\tau-\alpha) d \alpha
$$

Both sides take the Fourier transformation, and the result is:

$$
P_{s y}(w)=H(w) P_{y y}(w)
$$

Among them, $P_{y y}(w)$ stands for the power spectrum density of $y(t)$, and $P_{s y}(w)$ stands for the cross-power spectrum density of $s(t)$ and $y(t)$, And since $s(t)$ and $d(t)$ are uncorrelated, and then Formula (2.3) is satisfied 


$$
H(w)=\frac{P_{s}(w)}{P_{s}(w)+P_{d}(w)}
$$

As formula (2.3) shows us, signal all pass through when the noise is zero, noise is suppressed by all when the signal is zero. So the Weiner filter has the ability to filter out the noise whose speech enhancement algorithm ${ }^{[5]}$ is shows in Fig. $1^{\text {. }}$

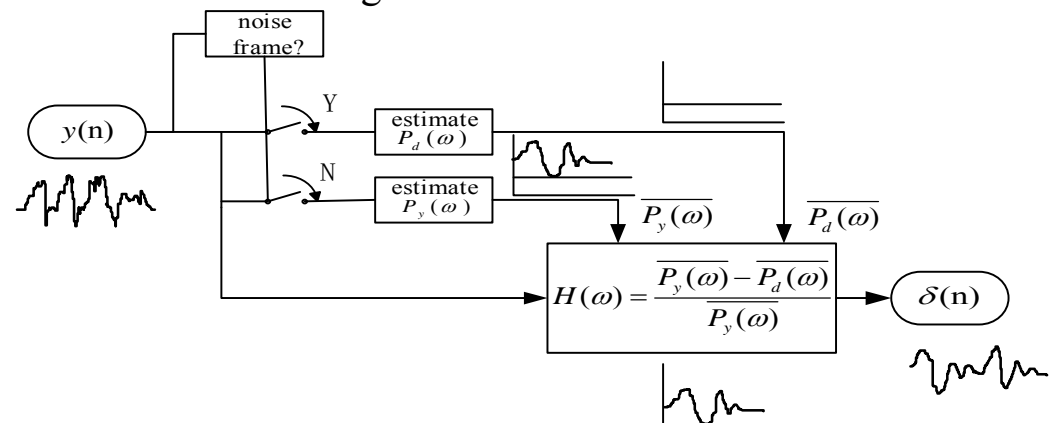

Fig. 1 The realization diagram of speech enhancement algorithm of Weiner filtering

The greatest advantage of using the Weiner filter ${ }^{[6]}$ is enhanced residual noise is similar to white noise, rather than undulating rhythm of musical noise.

\section{Iterated Weiner filter speech enhancement system}

\subsection{Iterated Weiner filter and its basic ideas}

Weiner filter can make the enhanced residual noise similar to white noise to improve voice quality of hearing. However, the residual background noise is serious, We can use an iterative Weiner filter to filter the background noise more effectively.

We do not stop updating the Weiner filter function and iterative filtering each singal until the speech enhancement effect and be increased significantly. We set a threshold to end the iteration, and output enhanced clean speech. Here is the Weiner basic schematic of improved filtering speech enhancement algorithm ${ }^{[7]}$. Shown in Fig. 2

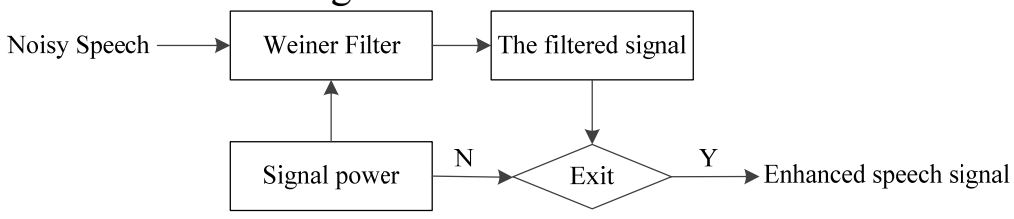

Fig. 2 The basic principle diagram of Iterated Weiner filtering

\subsection{Iterated Weiner filter speech enhancement algorithm}

Selectint a reasonable threshold indicators is important for speech enhancement algorithm implementation and effect, We selected the Euclidean distance in each frame of speech signal after two iterations LPC coefficients output voice as judged by an adjacent end of the iteration condition. The euclidean distance between $\alpha=\left(\alpha_{1}, \alpha_{2}, \cdots, \alpha_{n}\right)$ and $\beta=\left(\beta_{1}, \beta_{2}, \cdots, \beta_{n}\right)$ is defined as follows

$$
\text { dis } \tan c e(\alpha, \beta)=\sqrt{\sum_{i=1}^{n}\left(\alpha_{i}-\beta_{i}\right)^{2}}
$$

The given Weiner iteration process of each frame speech is as follow:

1.Providing suitable LPC coefficient euclidean distance threshold value as the basis of adjusting whether the iteration is ended or not

2.Initialization $P(w)_{0}=P_{y}(w) i=0, i$ is the iterations

3.Calculate:

$$
\begin{gathered}
H(w)_{i}=\frac{P_{s}(w)_{i}}{P_{s}(w)_{i}+\lambda_{d}(w)} \\
S(w)_{i+1}=H(w)_{i} Y(w) \\
P_{s}(w)_{i+1}=\left|S(w)_{i+1}\right|^{2}
\end{gathered}
$$




$$
i=i+1
$$

4.Repeating step 3 according to the condition of 1 until the end of the iteration to receive the enhanced voice frames

The flowchart of iteration Weiner filtering voice enhancement algorithm is shown in Fig. 3

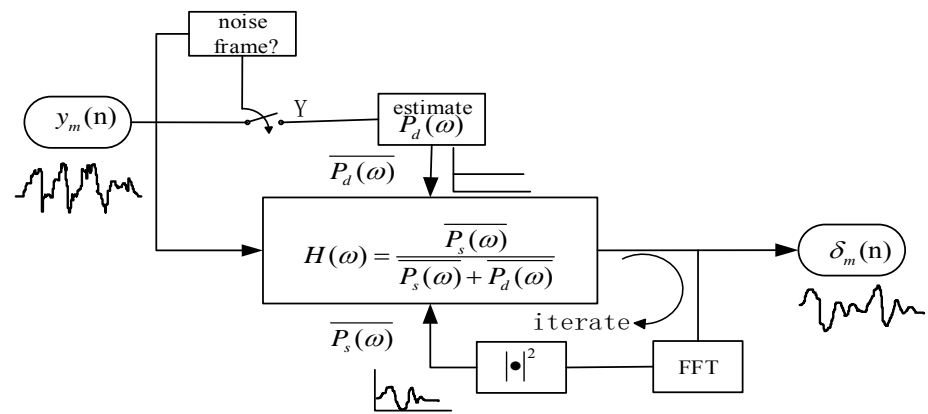

Fig. 3 The flowchart of iteration Weiner filtering voice enhancement algorithm

\section{Experiment and Analysis}

We will compare the enhancement of two enhancement algorithms on the signal to noise ratio, waveform, spectrogram and subjective hearing speech by giving the experimental data and analysis

\subsection{The selection of experimental parameters}

Adding white noise into pure speech signal to get noisy speech signal Here is speech parameters for both experiments as Table 1

Table 1 Experiment speech parameters

\begin{tabular}{cc}
\hline Parameter Name & Parameter values \\
\hline Noise power & $-32.762 \mathrm{~dB}$ \\
Sampling frequency & $8000 \mathrm{~Hz}$ \\
Sampling digit & $16 \mathrm{bit}$ \\
Frame size & 256 Sampling point/ frame (32 ms/frame) \\
Frame shift & 128 Sampling point \\
Windowing type & Rectangular window \\
Amplitude threshold value 1 & 14 \\
Amplitude threshold value 2 & 8 \\
Short-time zero-crossing rate & 20 \\
threshold value & \\
\hline
\end{tabular}

\subsection{Experimental results of Weiner filter}

In Weiner filter, we use double threshold determination method to conduct speech endpoint detection, according to the above set of experimental parameters, Weiner filtering shows the results of speech enhancement algorithm in Fig. 4

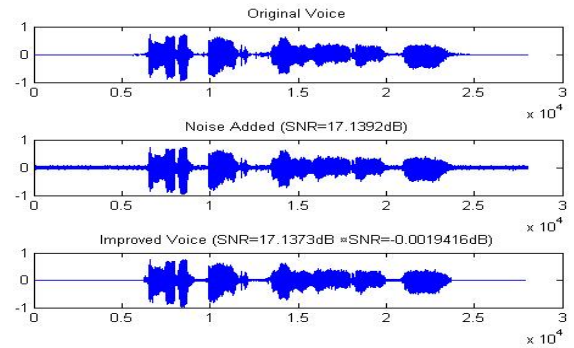

a)

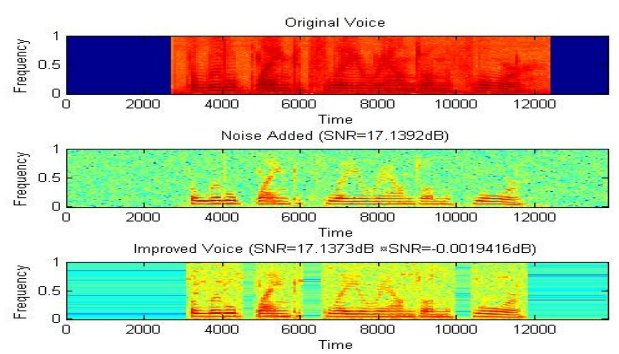

b)

a)Weiner filter's oscillograph of pure speech, noisy speech and enhanced speech

b) Weiner filter's speech spectrogram of pure speech, noisy speech and enhanced speech

Fig. 4

From Fig. 4a) contrast of waveform after Weiner filtering clean speech, noisy speech and enhanced voice, you can see the remaining spikes of enhanced voice waveform decreased. From Fig. 4b)spectrogram contrast, we can see there remains serious white noise in the background. Voice 
quality is still not satisfactory.

\subsection{Experimental results of Iterated Weiner filter}

In this experiment, we select the euclidean distance of LPC coefficients after two adjacent iterations as judgement for the end of the iteration. Settinh the threshold as 0.0036, whereby we can get the results of iteration Weiner filtering speech enhancement algorithm shown in Fig. 5.

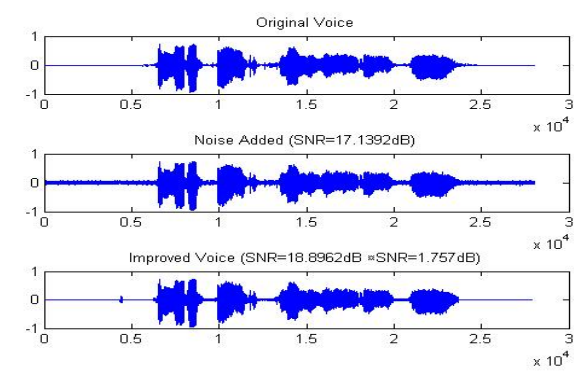

a)

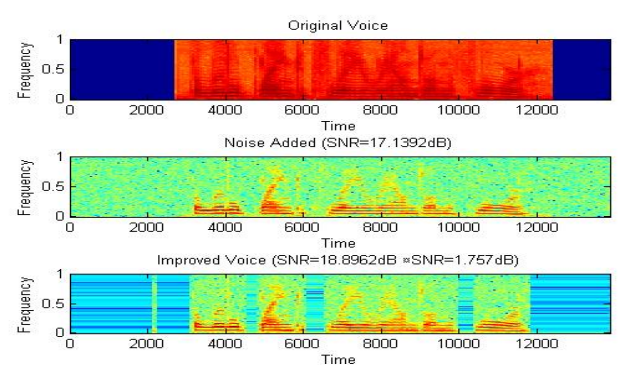

b)

a)IterationWeiner filter's oscillograph of pure speech, noisy speech and enhanced speech

b) IterationWeiner filter's speech spectrogram of pure speech, noisy speech and enhanced speech Fig. 5

From Fig. 5a) contrast of waveform after Weiner filtering clean speech, noisy speech and enhanced voice, you can see the remaining spikes of enhanced voice waveform amost disappear at all. From the comparison chart 5b) spectrogram of the spectrum, you can see the isolated points remaining in the enhancement speech also being filtered out. Aurally feel the filtered white noise background is relatively suppressed, musical noise almost disappeared, auditory quality with respect to the Weiner filter has been improved a lot.

\section{Conclusion}

Through the above experiments, we get the following conclusions: Although modified iterative Weiner filtering algorithm is relatively complex, and it is more difficult to realize than Weiner filtering algorithm, it can reduce more background white noise remained in the enhanced voice while eliminating the musical noise, and the speech enhancement effect is better than that of Weiner filtering algorithm.

\section{References}

[1] ChangLi. Fourth about processing of speech signal's current situation and Outlook. Physics, Vol.34(2005) No.4,p.300-306.

[2] Chen YongQuan, Zhang $\mathrm{Yu}, \mathrm{Hu}$ YongHao. Processing of speech signal technology and its application prospect. Network security technology and Application, Vol.36(2014) No.2,p.58-59.

[3] Yang Yi, Li ZeWei, Deng BeiXing. The reform and practice of processing of speech signal experiment. Laboratory research and exploration, Vol.33(2014) No.2,p. 123-126.

[4] Meng Jing, Xu Gang. Study on the evaluation of speech enhancement algorithm[J]. Computer Engineering, Vol.32(2006) No.24,p. 223-225.

[5] Bai WenYa, Huang JianQun, Chen ZhiLing. Improvement of speech enhancement algorithm based on Wiener filtering.Electro acoustic technology,Vol.31(2007) No 1,p 44-46.

[6] Mukul Bhatnagar, B.E. A modified Spectral Subtraction method comband with Perceptual weighting for speech enhancement,The University of Texas at Dallas,2002.

[7] Ding QianJun, Wang YongLiang, Zhang YongShun. A quick implementation algorithm of the multi-stage wiener filter, iterative related subtraction a lgorithm[J]. Journal of Communications, Vol.26 (2005) No.12,p. 1-7. 